were used to collect views on stakeholders' perception of benefits, opportunities and challenges of harmonisation.

Results Sixty-nine (69) accredited RECs were mapped. All countries had national ethics guidelines and National Research Regulatory Authorities, whose mandates varied across countries. $57 \%$ of RECs reviewed local and international research, $43 \%$ reviewed local studies only. On average, 91 protocols were reviewed annually across all RECs (range 15 to 200). Membership ranged from 6 to 22 members per REC, with age range of 29 to 75 years.

Annual budget allocation ranged from $\$ 3000$ to $\$ 2.9$ million financed through review fees (84\%) or/and institutional budget (14\%). $71 \%$ of RECs had education policy but $41 \%$ had members with training in ethics. Review turn-around time ranged from 14 to 90 days. All RECs supported harmonisation and attributed it to improved efficiency, quality and standardised costs.

Conclusion Similarities and dissimilarities were noted in the EAC countries' ethics review frameworks. Harmonisation should consider 1) harmonisation of policy frameworks and tools; 2) institutionalisation of regional joint review mechanisms, 3) standardisation of training and capacity strengthening, 4) Review of the REC operational and financing models.

\section{OC 8477 MAKING RESEARCH EASIER, BETTER AND FASTER THROUGH KNOWLEDGE SHARING AND BUILDING COMMUNITIES OF PRACTICE}

Leandro Abade. The Global Health Network, UK

\subsection{6/bmjgh-2019-EDC.21}

Background The Global Health Network improves the health research capacity of low- and middle-income countries (LMICs) by facilitating know-how and knowledge exchange between organisations, disease areas, regions and roles. We show how we use the sharing phenomenon to transform and drive health research in resource-limited settings.

Methods We harnessed cutting-edge technology that facilitates engagement of medical researchers and healthcare professionals in low-resource settings and creates opportunity for knowledge exchange and capacity development in global health. We built highly functional digital communities of practice (CoPs) that serve as learning and sharing tools for researchers, and a gateway for sharing experience about disease research. These CoPs are intertwined with regional activities such as training workshops with health care professionals, which helps to enhance engagement and impact.

Results We disseminate resources with collaborators to enable research in LMICs via 37 CoPs. The data derived from these CoPs is used to identify knowledge-gaps in research capacity, as well as a guide to the development of resources and tools, such as standardised outcome measures. The network has developed 25 free peer-reviewed e-learning courses covering a wide range of topics, as well as high-quality professional development tools. We highlight the WHO-TDR Professional Development Scheme that tracks and guides training and career development. The network has trained over 33500 professionals (with $>400 \mathrm{k}$ courses taken) across 119 LMICs on the principles of good clinical practices ensuring the safety of research participants and the integrity and validity of research data.

Conclusion The Global Health Network is enabling research in areas where data is missing by providing tools, training, and resources whilst supporting and training research teams. The next step is to widen this impact and invite new researchers to drive and enable more and better clinical research in places and situations where evidence is still woefully lacking.

\section{OC 8489 CLINICAL DEVELOPMENT OF A THERAPEUTIC VACCINE FOR PREVENTION OF POST KALA AZAR DERMAL LEISHMANIASIS}

${ }^{1}$ Paul Kaye, ${ }^{2}$ Ahmed Musa*, ${ }^{6}$ Joseph Olobo, ${ }^{5}$ Margaret Mbuchi, ${ }^{3}$ Asrat Hailu Mekuria, ${ }^{4}$ Flavia D'Alessio, ${ }^{4}$ Sophie Houard, ${ }^{4}$ Odile Leroy. ${ }^{1}$ University of York, UK; ${ }^{2}$ IEND, University of Khartoum, Sudan; ${ }^{3}$ University of Gondar, Ethiopia; ${ }^{4}$ European Vaccine Initiative, Universitäts Klinikum Heidelberg, Germany; ${ }^{5}$ Kenya Medical Research Institute, Nairobi, Kenya; ${ }^{6}$ Makerere University, Kampala, Uganda

\subsection{6/bmjgh-2019-EDC.22}

Background The leishmaniases represent a complex of human diseases, with 350 million people at risk of infection worldwide. Although the potential benefits of vaccination have been well-recognised, no human vaccine is registered. Post-kala azar dermal leishmaniasis (PKDL) is a chronic skin disease often following treatment for visceral leishmaniasis (VL). In addition to affecting quality of life, evidence suggests that PKDL patients may also act as reservoirs for VL transmission. Hence, PKDL vaccines may have a significant impact on disease burden. We recently developed a third-generation adenoviral vaccine for leishmaniasis (ChAd63-KH) that has been evaluated for safety and immunogenicity in healthy volunteers (Osman et al,2017). ChAd63-KH is currently being evaluated for safety as a therapeutic in Sudanese PKDL patients, with a phase IIb RCT starting in late 2018. With EDCTP funding, we are initiating a new phase IIa/IIb study (PREV_PKDL) to determine whether ChAd63-KH can prevent PKDL development.

Methods In PREV PKDL, we will conduct an open-label phase IIa safety study, followed by a placebo blinded, phase IIb RCT. Safety and clinical response represent primary outcome measures, and immunogenicity is a secondary outcome measure. In addition, working across the four countries of Leishmaniasis East Africa Platform (LEAP), we will use deep phenotyping methods to study the immune status of patients before and after treatment for VL to understand why PKDL development is limited to specific geographic regions. This work, and other research in the region, will be supported by the creation of a new flow cytometry 'centre of excellence' within LEAP.

Results An update on the progress of our current therapeutic trial in PKDL patients will be provided.

Conclusion PREV_PKDL represents an important step in the clinical development of ChAd63-KH and will help develop capacity to support future vaccine and drug trials for leishmaniasis in the East Africa Region. 